\title{
Voxelotor Modulates the Analgesic Effect of Certain Opioids
}

\author{
Samir K. Ballas
}

\section{To the Editor}

Voxelotor (Oxbryta, Global Blood Therapeutics (GBT) 440) was developed by GBT for the treatment of sickle cell disease (SCD) in adults and children 12 years of age and older. It exerts its action by binding to the amino acid terminal of both $\alpha$ chains of hemoglobin ( $\mathrm{Hb}$ ). This, in turn, prevents or decreases the polymerization of hemoglobin $\mathrm{S}(\mathrm{HbS})$ by increasing $\mathrm{Hb}$ oxygen affinity. The efficacy and safety of voxelotor in SCD was evaluated in a phase III randomized, double-blind, placebo-controlled multicenter trial in combination with and without Hounsfield unit (HU) (Heart Outcome Prevention Evaluation Trial (HOPE Trial)) [1]. It was approved by the United States Food and Drug Administration on November 19, 2019 [2]. The approval was accelerated based on increase in $\mathrm{Hb}$. Continued approval for this indication may be contingent upon verification and description of clinical benefit in confirmatory $\operatorname{trial}(\mathrm{s})$.

Efficacy was based on $\mathrm{Hb}$ response rate defined as $\mathrm{Hb}$ increase of $>1 \mathrm{~g} / \mathrm{dL}$ from baseline to week 24 in patients treated with voxelotor $1,500 \mathrm{mg}$ versus placebo. The response rate for voxelotor $1,500 \mathrm{mg}$ was $51.1 \%$ (46/90) compared to $6.5 \%$ $(6 / 92)$ in the placebo group $(\mathrm{P}<0.001)$.

Recommended dosage of voxelotor is $1,500 \mathrm{mg}$ orally once daily with or without food. Recommended dosage for severe hepatic impairment is $1,000 \mathrm{mg}$ orally once daily with or without food [2].

From the pharmacokinetic point of view, in vitro and in vivo studies showed that voxelotor is excessively metabolized through phase I metabolism that includes the utilization of cytochrome P450 (CYP450) enzymes (oxidation and reduction), phase II (glucuronidation) and a combination of both phases. Oxidation of voxelotor is mediated mostly by CYP3A4 enzyme with minor contributions from CYP2CI9, CYP2B6 and CYP2C9 [2]. This multiplicity of metabolic pathways renders voxelotor vulnerable to drug-drug interactions where other dugs affect its metabolism or, conversely, where voxelotor

Manuscript submitted October 27, 2020, accepted November 18, 2020

Published online February 25, 2021

Cardeza Foundation for Hematologic Research, Department of Medicine, Sidney Kimmel Medical College, Thomas Jefferson University, 1020 Locust St, Suite 390, Philadelphia, PA 19107, USA. Email: samir.ballas@jefferson.edu

doi: https://doi.org/10.14740/jocmr4384 itself affects the metabolism of other drugs. Thus, the daily dose of voxelotor has to be adjusted in the presence of strong CYP3A4 inhibitors, to $1,000 \mathrm{mg}$ once daily because inhibition of CYP3A4 maintains a relatively higher concentration of voxelotor in the blood. Conversely, inducers of CYP3A4 result in rapid degradation of voxelotor into inactive metabolites that require increasing the dose to $2,500 \mathrm{mg}$ once daily [2]. Potent inducers of CYP3A4 that may be prescribed to patients with SCD include barbiturates, carbamazepine, phenytoin, dexamethasone and rifampin. Potent inhibitors of CYP3A4 often prescribed to patients with SCD include cimetidine, fluoroquinolones, selective serotonin reuptake inhibitors, quinine, macrolides and calcium-channel blockers (diltiazem).

The plot thickens if the metabolic profile of opioids is brought into the picture. The significance of the role of opioids to manage the pain of the acute painful vaso-occlusive crisis (VOCs) becomes evident from the recent advances in understanding the pharmacogenomics of drugs in general including the opioids [3]. The metabolism of certain opioids also includes phase I pathway shown in Table 1. Phase I involves the CYP450 enzymes whereas glucuronidation is the major metabolic pathway in phase II metabolism. Phase I metabolism of opioids involves primarily the CYP3A4 and CYP2D6 enzymes. The CYP3A4 enzyme metabolizes more than 50\% of all drugs; consequently, opioids metabolized by this enzyme have a high risk of drug-drug interactions [4]. The CYP2D6 enzyme metabolizes fewer drugs and is therefore associated with an intermediate risk of drug-drug interactions [4]. The CYP3A4 enzyme is the primary metabolizer of fentanyl and oxycodone, although normally some oxycodone undergoes CYP2D6-mediated metabolism to oxymorphone (Table 1) [4]. Tramadol undergoes both CYP3A4-mediated and CYP2D6mediated metabolism. Methadone is primarily metabolized by CYP3A4 and CYP2B6. CYP2C8, CYP2CD19, CYP2D6, and CYP2C9 also contribute in variable degrees to its metabolism [5]. Since voxelotor itself inhibits CYP3A4, the co-administration of certain opioids (hydrocodone, oxycodone, fentanyl and methadone) shown in Table 1 will increase their analgesic effect and/or their side effects especially after multiple dosing. Figure 1 shows an example of the effect of inhibiting the CYP3A4 enzyme on the analgesic efficacy of fentanyl. The same applies to oxycodone, methadone and tramadol.

The plot thickens further considering the complexity of the pharmacogenomics of the CYP450 system where the enzymes may be deleted, mutated, duplicated or even triplicated [6]. Human CYP consists of 21 currently described families and 20 subfamilies coded by 57 genes and 58 pseudogenes [7]. 
Table 1. Metabolism of Certain Opioids Used in Sickle Cell Disease by the Cytochrome P450 Enzymes

\begin{tabular}{lll}
\hline Opioid & CYP450 enzyme & Active metabolite \\
\hline Codeine & CYP2D6 & Morphine, hydrocodone \\
Hydrocodone & CYP2D6, 3A4 & Hydromorphone \\
Oxycodone & CYP2D6, 3A4 & Oxymorphone \\
Fentanyl & CYP3A4 & None \\
Methadone & CYP2D6, 3A4, 2C8, 2C9, 2C19,2B6, 1A2 & None \\
Tramadol & CYP2D6, 3A4, 2B6 & None \\
\hline
\end{tabular}

Adapted from Ballas S. K. Sickle Cell Pain, Second Edition. Washington DC: International Association for the Study of Pain; 2014. CYP450: cytochrome P450.

The CYP isoforms 1, 2, and 3 are responsible for the majority of hepatic drug metabolism. Of these, CYP2C9 and CYP2D6 are involved in the metabolism of several drugs used for pain control including opioid and nonopioid analgesics. Phase I metabolism of opioids involves primarily the CYP3A4 and CYP2D6 enzymes. The CYP3A4 enzyme metabolizes more than $50 \%$ of all drugs; consequently, opioids metabolized by this enzyme have a high risk of drug-drug interactions. Accordingly, medications used in addition to opioids may enhance or inhibit the metabolism of the opioid in question.

In the phase III trial of voxelotor [1], a VOC during treatment was defined, among other things, as pain that required treatment with oral or parental opioids, ketorolac, or other analgesics. Usually opioids are used routinely in most cases. However, the specific opioids used were not mentioned. Thus, we do not know if the opioids used belonged to phase I CYP450 or to phase II glucuronidation. This might have created a confounding factor.

Thus, if the voxelotor group received more opioids metabolized by CYP3A4 shown in Table 1, the chances are that they may have achieved better pain control than the placebo group and hence, requiring less intake of more opioids. The voxelotor (HOPE) trial did not mention the amount of opioid intake by the groups involved in the study.
If the voxelotor group did indeed consume fewer opioids, this would have been considered a positive clinical response. Future trials should consider the specific opioids used especially if the drug being studied inhibits certain CYP450 enzymes shown in Table 1. It is highly recommended that future such studies limit the use of opioids to opioids phase II drugs such as morphine or hydromorphone.

\section{Acknowledgments}

None to declare.

\section{Financial Disclosure}

None to declare.

\section{Conflict of Interest}

Member of Novartis Speakers Bureau and Consultant for Novartis and Global Blood Therapeutics (GBT). However, GBT did not have any input in the design, format, or construc-

A.

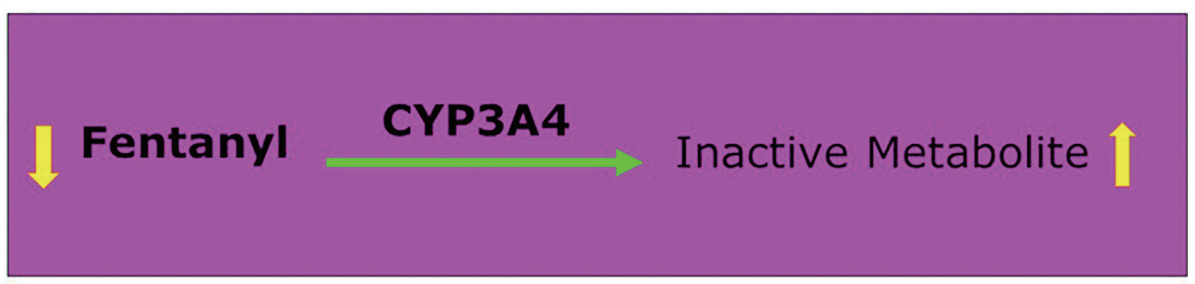

B.

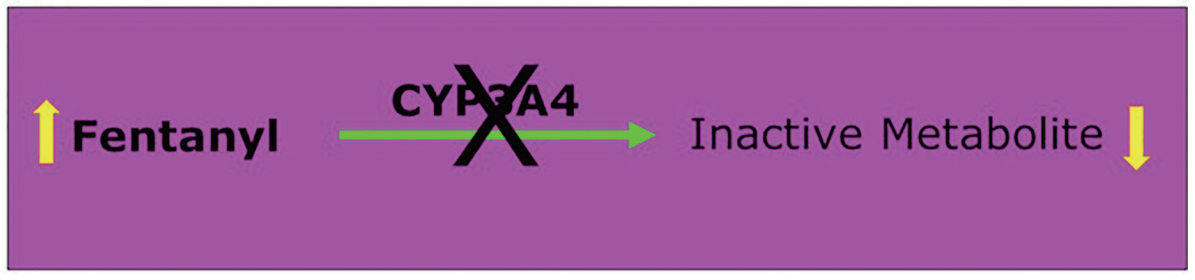

Figure 1. Effect of CYP3A4 enzyme on the metabolism of fentanyl. Equation A shows how CYP3A4 enzyme usually metabolizes fentanyl by oxidizing it to inactive metabolites and hence, decreases its concentration in blood. Equation $B$ shows that inhibition of CYP3A4 decreases the oxidation of fentanyl to inactive metabolites and hence, maintains its concentration in blood to maintain or increase its analgesic effect. 
tion of this manuscript.

\section{Informed Consent}

Not applicable.

\section{Author Contributions}

Dr. Ballas has contributed solely and in whole to this report.

\section{Data Availability}

The author declares that data supporting the findings of this study are available within the article.

\section{References}

1. Vichinsky E, Hoppe CC, Ataga KI, Ware RE, Nduba V,
El-Beshlawy A, Hassab $\mathrm{H}$, et al. A phase 3 randomized trial of voxelotor in sickle cell disease. N Engl J Med. 2019;381(6):509-519.

2. Global Blood Therapeutics. OXBRYTA (voxelotor) tablets [Package insert]. San Francisco, CA.; 2019.

3. Ballas SK. Chapter 11: pharmacogenetics, genetics, epigenetics, and genetic markers of sickle cell pain. Sickle Cell Pain, Second Edition. Washington DC: International Association for the Study of Pain; 2014. p. 391-407.

4. Smith HS. Opioid metabolism. Mayo Clin Proc. 2009; 84(7):613-624.

5. Totah RA, Allen KE, Sheffels P, Whittington D, Kharasch ED. Enantiomeric metabolic interactions and stereoselective human methadone metabolism. J Pharmacol Exp Ther. 2007;321(1):389-399.

6. Polasek TM, Lin FP, Miners JO, Doogue MP. Perpetrators of pharmacokinetic drug-drug interactions arising from altered cytochrome P450 activity: a criteria-based assessment. Br J Clin Pharmacol. 2011;71(5):727-736.

7. Zhou SF, Liu JP, Chowbay B. Polymorphism of human cytochrome P450 enzymes and its clinical impact. Drug Metab Rev. 2009;41(2):89-295. 